Meta

Journal des traducteurs

Translators' Journal

\title{
La traduction comme OVNI
}

\section{Alexis Nouss}

Volume 40, numéro 3, septembre 1995

La traduction, qu'est-ce à dire? Phénoménologies de la traduction

URI : https://id.erudit.org/iderudit/003748ar

DOI : https://doi.org/10.7202/003748ar

Aller au sommaire du numéro

Éditeur(s)

Les Presses de l'Université de Montréal

ISSN

0026-0452 (imprimé)

1492-1421 (numérique)

Découvrir la revue

Citer cet article

Nouss, A. (1995). La traduction comme OVNI. Meta, 40(3), 335-342.

https://doi.org/10.7202/003748ar

\section{Résumé de l'article}

In view of the shaky foundations of translation studies as a discipline, the author suggests an hermeneutic epistemology of translation. Of two possible approaches, which are described using the analogy of the "water diviner" (sourcier) and "well maker" (puisatier), the author opts for the former, and justifies his choice by defining translation as a transformation process, a primary, not secondary, language operation. He cites contemporary literary and philosophical theories to look at the issues of identity and alterity in translation, as well as the role of the history and the uncertainty of meaning. According to Nouss, translation is in essence an always Unidentifiable Flying Object (UFO).
Ce document est protégé par la loi sur le droit d'auteur. L'utilisation des services d’Érudit (y compris la reproduction) est assujettie à sa politique d'utilisation que vous pouvez consulter en ligne.

https://apropos.erudit.org/fr/usagers/politique-dutilisation/ 


\title{
LA TRADUCTION COMME OVNI
}

ALEXIS NousS

Universite de Montreal, Montréal, Canada

\begin{abstract}
Résumé
In view of the shaky foundations of translation studies as a discipline, the author suggests an hermeneutic epistemology of translation. Of two possible approaches, which are described using the analogy of the "water diviner" (sourcier) and "n'ell maker" (puisatier). the author opts for the former, and justifies his choice by defining translation as a iransformation process, a primary, not secondary, language operation. He cites contemporary literary and philosophical theories to look at the issues of identity and alterity in translation, as well as the role of the history and the uncertainty of meaning. According oo Nouss, translation is in essence an always Unidentifiable Flying Objert (UFO).
\end{abstract}

Étrange objet que ce numéro, dira le lecteur le parcourant d'un premier regard : un entretien, un extrait de lettre, des aphorismes, une taxinomie, une nouvelle même, à côté, certes, d'articles de facture plus ou moins traditionnelle. Quant à mes invités, si certains $s$ "inscrivent naturellement dans le champ traductologique en ayant déjà produit dans le domaine, d'autres proviennent de disciplines for diverses. L'auberge du lointain. pour reprendre l'expression d'Antoine Berman, paraît vouée à la gastronomie ibérique.

Et le sous-titre ? Phénoménologies : au pluriel ? Et que nous chante donc le titre ? Qu'y-a-t-il à dire de la traduction qu'on ne sache déjà ?

Précisément : que sait-on de la traduction ? Voilà bien un étrange objet (épistémologique). À dire vrai, elle me semble avoir tout d'un objet non identifié, et même volant, OVNI à part entière : parcourant les disciplines, les effleurant sans jamais s'y poser, sauf à y être obligée pour des raisons idéologiques. Objet fantôme, donc. Elle hante les théories, elle apparaît çà et là dans divers champs du savoir : linguistique, sémiotique. littérature comparée. psychanalyse, anthropologie, philosophie, d'autres encore, chacun y allant de sa spéculation et de sa définition, dans un joyeux désordre conceptuel où l'utilitarisme fait force de loi. Dynamique langagière fondée sur l'écart, la distance, l'absence, la perte, faut-il se résoudre à ce que la pensée de la traduction soit aussi soumise à une règle d'indétermination? Peut-être prouve-t-elle ainsi sa place et son rôle paradigmatique dans l'épistémè de notre modernité. Il est tentant de poser qu'après la conscience au $\mathrm{XIX}^{\mathrm{e}}$ siècle et le langage au début du $\mathrm{XX}^{\mathrm{c}}$, la traduction s'affirme désormais comme ce qui permet de définir l'ethos contemporain, fondé sur l'échange et la communication'. Je m'y résoudrai si certains événements de l'actualité ne me prouvaient le contraire. Mais admettons l'hypothèse. étayée par la place centrale qu'occupe la traduction dans notre horizon philosophique, de Freud à Derrida.

Il s'agit donc de poser la question de la légitimité d'un domaine du savoir, d'une théorie de la connaissance centrée sur la traduction. La traductologie possède un nom. certes, mais quelle est sa réalité ? Par-delà le foisonnement des associations, publications, colloques et programmes universitaires, y-a-t-il véritablement là un champ disciplinaire, et comment s'est-il constitué ?

Au demeurant, la traduction peut-elle susciter un savoir, normatif et axiologique, de type traditionnel ? N'appelle-t-elle pas plutôt une épistémologie novatrice, transversale et transgressive? 
On répète à satiété que depuis qu'existe la pratique de la traduction, la théorie l’a accompagnée. Peut-être, surtout si l'on confond théorie et méthode, analyse et recette. pensée et système, commentaire et exégèse, critique et idéologic.

Le lecteur sera inévitablement frappé par le foisonnement divergent des ouvrages dans le domaine, se perdra dans l'absence de cohésion et le manque de rigueur conceptuelle, à croire que chaque auteur traite de son propre objet (la traduction comme résultat, comme production. comme processus. comme texte, etc.) sans que soient ressenties la nécessité d'un accord minimal sur quelques traits communs à l'objet traité ni l'honnêteté d'un positionnement idéologique ou historique. On ne se rassurera que précairement en avançant qu'il en est du Zeitgeist, ce prétendu post-modernisme si fragile qui croit, par l'amalgame, réguler la complexité.

Une étape semble avoir été omise, l'interrogation principielle : de quoi parle-t-on ? J'ose croire qu'il n'est pas trop tard el me propose de recueillir dans ce numéro des réponses à cette question, qui s'articuleront selon divers énoncés?. L’hétéroclite et l'hybride de l'ensemble, loin d’infirmer la validité de la tentative, sont recherchés comme des effets de la problématique abordée, sans toutefois, je l'espère, succomber à l'esthétique facile de l'objet fuyant qui exige une approche floue, tactique prémentionnée tant employée en nos jours de crise axiologique.

Je voudrais ici développer succinctement pourquoi la traduction appelle à mes yeux une telle théorisation flottante (au sens de l'attention demandée à l'écoute psychanalytique qui. on le sait, est métaphorisée par Freud comme forme de traduction) et donc quelle en serait ma phénoménologie?. L'histoire de la traduction est la traduction de l'histoire, ai-je développé ailleurs, mais un tel argument qui entend définir une herméneutique de la traduction demande à être précisé. Il est deux manières de comprendre l’herméneutique. Je les métaphoriserai — rhétorique habituelle en traductologie, étymologie oblige. métaphore renvoyant à transport, à transfert - par les figures du sourcier et du puisatiert. Ce dernier va creuser afin de faire jaillir le tlot attendu : le premier se contente d'indiquer le lieu d'un possible jaillissement. L'intérêt de cette représentation tient évidemment aussi dans le renouvellement quelle apporte au couple symbolique sourcier/cibliste, quelque peu surexploité.

La possibilité d’articuler ces deux positions herméneutiques m'est apparue lors d'un travail sur la poétique - d'écriture el de traduction. les deux ne faisant qu une de Paul Celan' dont l'importance n'est plus à démontrer dans la littérature de l'aprèsguerre. Pour interpréter son ceuvre poétique, certes difficile et opaque, deux courants d’interprétation s'opposent. Le premier postule la nécessité d'une connaissance externe. la plus complète possible, des données biographiques, historiques el encyclopédiques ayant présidé a l'écriture des poèmes. Le second table sur la poéticité propre au texte et sur son pouvoir d'évocation interne agissant sur la sensibilité subjective du lecteur. Le parallèle est aisé à établir avec deux courants en matière de traductologice : less tenants d'un sens assignable traversant le medium textuel et les défenseurs d'une signifiance en mouvement (celui du sujet et celui de l'histoire) attachée à la lettre du texte.

Or l’importance de la poétique celanienne, le lien qu'elle établit entre pesésie et traduction, n'est pas liée à la biographie propre de Celan qui unit à son activité de poète une immense production de traducteur (deux volumes sur les cinq de ses auves complètes). Elle ressort du lien profond existant naturellement entre poésie el traduction : "Language is set in motion in many ways, but no relations to language do so more than those named translation and poetry. $[\ldots \mid$ Experiencing language as language $|\ldots|$ is the special effort that links the poet and the translatorm. écrit Denis J. Schmidt (1990: 4-5), citant le geste de Benjamin et Celan pour redonner au langage sa capacité de mémoire et de rédemption. Il n'est, pour comprendre encore ce lien, que de faire référence à la réflexion d'Henri 
Meschonnic sur le rythme, seule mesure de l'histoire et du sujet, seul facteur de signifiance, contre le dualisme rigide et réducteur du signe. Sa pensée de la traduction comme "rapport demeuré rapport". et non transport', est à rapprocher de la troublante définition proposée par Char (que traduisit — on ne s'en étonnera guère - Celan) : «Le poème est l'amour réalisé du désir demeuré désir» («Partage formel» dans Seuls demeurent).

On peut voir aussi ce lien exprimé par une dynamique commune de transformation? : poésie et traduction transforment le langage comme ils transforment le sujet. auteur ou lecteur, saisi dans cette opération. Pour l'une et l'autre, le sens de l'énoncé tient dans l'énonciation et non dans une signification autonome et extérieure, il est à trouver dans le mouvement même du langage ${ }^{\not}$. évoqué plus haut, dans le devenir qu'il implique et qu'il manifeste. La phénoménologie de la traduction s'expérimente comme transformation et cette vision nous invite à abandonner les idées de parcours, et même de passage, habituellement utilisées (ce dont témoignent les notions de départ et d'arrivée pour désigner les langues ou les textes en jeu), ainsi qu'à réviser les raisonnements fondés sur des postulats d'équivalence, de compensation ou de fidélité. C'est le sens de la citation de Walter Benjamin : «La traduction est le transfert d'une langue dans une autre à travers un continuum de métamorphoses. La traduction traverse des continuums de métamorphose. non pas des zones abstraites d'équivalence et de ressemblance.» ( «Sur le langage en général et sur le langage humain" ${ }^{9}$ : ma trad.)

Le rôle de la traduction en est radicalement changé dans une pensée linguistique : elle n'est plus un processus second et dérivé dans l'exercice langagier, elle en devient le principe. comme le développèrent Benjamin puis George Steiner, posant l'équation entre textualité et traduisibilité. On ne pensera plus la traduction à partir du langage mais, dès lors, le langage à partir de la traduction ${ }^{\circ}$. S'ouvre ainsi l'espace d'une «Critique de la traduction 11 " qui fait de celle-ci un horizon herméneutique essentiel à la saisie du monde el de l'histoire modernes vécus sous les espèces de l'incertitude et de l'auto-fondation'12.

D'où son aisance à être pensée dans le paysage de la théorie littéraire des demières décennies. Le passage de l'cuuvre au texte. dissipant l'ombre d'une pesante et trop présente patemité, rend a la traduction la liberté et le plaisir d'une réćcriture sans culpabilité, ce qu'elle connaissait dans les siècles passés, d'une économie scripturaire ignorante des limites génériques et linguistiques (Berman 1988) - comme quoi, soit dit en passant, la modernité n'a rien d'une notion chronologique. La mort de l'auteur signifie. en somme, la naissance du traducteur et les réflexions de Barthes ou Foucault sur le "plaisir du texte» ou sur la «fonction-auteur» gagneraient à être relues en songeant à l'objet «traduction». De même, il n'est plus besoin d'indiquer combien l'œuvre de Derrida - outre ses réflexions traitant directement de la traduction, thème majeur de sa pensée - offre d'éléments fertiles susceptibles de renouveler l'analyse du processus traductif : la differance, le supplément. le «double bind", l'absence d'origine, etc.

Par ailleurs, la traduction, comme trans-action sémiotique et sémantique, s'offre comme modèle et illustration de ce que l'on sait désormais des rapports entre identité et altérité. L'idéologie traditionnelle posait dans une perspective essentialiste et homogénéisante des territoires culturels auxquels la traduction servirait de medium de communication, uni- ou bilatéral, voire d'échange ${ }^{13}$. Or la traduction, en résonance avec la pensée contemporaine, sert précisément à dénoncer toute prétention de ce type quant aux fondations et aux limitations des langues et des cultures ${ }^{14}$. La traduction est une subversion dont la fonction déstabilisatrice redécoupe la figure de l'étranger. Les polémiques stériles sur l'appropriation culturelle pourraient en tirer un solide enseignement. Certes, selon l'adage répété à satiété, "Traduttore traditore» ( «le traducteur est serviteur de deux maîtres", disait encore Franz Rosenzweig) mais une trahison peut être noble, cela dépend de la cause, tout comme une transgression. Et quant à choisir une image de duplicité (au 
sens étymologique), je préfère à celle du traitre celle du rival. pour reprendre deux catégories familières au XVIII" siècle (voir les opéras de Mozart).

La traduction était idéologiquement comprise dans la dynamique du même et de l'autre. Traduire serait convertir de l'autre en même, le faire écrire comme si il (elle) avait écrit dans la langue d'arrivée. Mais l'altérité n'est jamais à sens unique. L'autre ne renvoie pas au même mais à un autre autre, sujet toujours changeant dans sa discursivité et son historicité. "La traduction est celte activité qui permet mieux qu aucune autre, puisque son lieu n'est pas un terme mais la relation elle-même, de reconnañtre une altérité dans une identité" écrit Meschonnic (1992: 106). De Levinas à Ricour. de Foucault à Lyotard, le faux procès de l'identité et de l'altérité, du soi et de l'autre, a été définitivement jugé.

En termes marxistes 15 , la traduction relève d'une valeur d'usage, non d'échange. Et ainsi que le montraient les travaux du groupe Tel Quel, reconnaître la valeur d'usage d'un texte (ou d'un signe ou d'une langue ou d'une traduction). c'est admettre qu il est un moyen de production, susceptible d'autonomie et de création, et non une marchandise. $n$ 'ayant de fonction que communicante. La traduction est par excellence ce qui peut susciter la multiplicité textuelle et chacun de ses produits aura sa valeur en soi. et non au regard d'intérêts ou d'investissements extérieurs. Elle ne véhicule pas de "valeurs", étrangères ou autochtones : elle est à elle-même sa propre valeur puisqu'elle est un texte ( «traduction-lexte" dit Meschonnic). Elle est, sur le plan textuel, l'équivalent de l'identité sur le plan ontologique, entre le même et l'autre, ni le même ni l'autre : elle est, plus qu' ${ }^{\prime}$ 'n devenir, un devenir, un dire qui ne s'effacerait jamais devant le dit qu'il produit et qui le manifeste. C'est cette tension inhérente à la nature de la traduction qui explique fondamentalement le fait admis des (re)traductions multiples d'un même original et non les seules variations linguistiques ou socioculturelles.

Commentant un propos de Nietzsche sur la permanence du devenir qui fait que nous appelons faussement arbre une forme au constant mouvement de laquelle nous sommes aveugles, Roland Barthes écrit : "Le Texte serait lui aussi cet arbre dont nous devons la nomination (provisoire) à la grossièreté de nos organes. Nous serions scientifiques par manque de subtilité." (1973: 96). Celle «science du devenir" qui pourrait seule ne pas rebuter le "plaisir du texte" et que Barthes appelait de ses voux, j'oserai croire qu'elle s'annonce sous les espèces de la traductologie. De fait. si le texte en général ressemblait à cet arbre nietzschéen, le texte traducrif lui ressemblerait encore davantage. Ce signifiant néologique que j’introduis ici le prouve assurément. Que seraitil ? Il désignerait une forme textuelle en mouvement, en tension, une textualité dialectique qui unirait l'original et sa traduction sans sacrifier l'un ou l'autre. l'un à l'autre, sollicitant le "rapport demeuré rapport" prémentionné. Mais ce déséquilibre structurant - et nous sommes proches ici des théories du chaos et du désordre. des logiques de l'incertitude et de la complexité qu'aussi bien Edgar Morin qu'Henri Atlan ont interrogées - pose un problème épistémologique que la théorie littéraire, par exemple, crut résoudre il y a quelques décennies en optant pour le structuralisme ou la sémiologie et en substituant le texte à l'ocuvre. Comment en effet cerner ce phénomène fuyant, plus encore : dont la fuite est l'essence?

Il n'est pas dans la visée du présent numéro de traiter d'épistémologie, aussi ne ferai-je que préciser brièvement comment la phénoménologie ci-dessus décrite suggère cette approche que je désigne comme une herméneutique de la traduction et que je définirais sous la figure du sourcier et non du puisatier. non la recherche d'un sens figé à découvrir mais la quête d'une signifiance soumise à l'historicité el à l'individualité du sujet traducteur. L'herméneutique s'oppose alors à la linguistique : celle-ci s'appuie sur la langue, matériau solide, décomposable, grammatifiable: la première traite de l'histoire. 
fluide, octanique, polysémique. La linguistique, visant la certitude, s'occupe de signification ; l'herméneutique, jouant de l'incertitude, s'occupe d'interprétation.

Une tarte à la crème traductologique pose la question de l'apparentement de la traduction à l'art ou à la science, séparant les défenseurs à tout crin de la subjectivité traductive des tenants d'une prétention objectivante. Fausse question, car elle $n$ 'interroge pas les presupposés qui fonderaient ces deux directions ainsi proposées à la pratique traductionnelle. Le vrai questionnement devrait plutôt porter sur la réception du texte à traduire et rechercher si elle se rapproche de l'interprétation esthétique, de la recherche explicative scientifique, ou si elle ne développe pas plutôt une forme originale d'hybridité herméneutique entre les deux positions, annonçant la nouveauté paradigmatique de la traduction dans l'épistémé contemporaine. C'est notamment le statut de l'agent, créateur ou observateur, qui est en question. Dans quelle mesure participe-t-il au processus, et l'influence-t-il ? Expliquant le rôle central de l'herméneutique dans le paysage philosophique contemporain par son insistance sur "l'instance éthique», Vattimo écrit : «En tant qu'ethos, coutume. culture partagée par une époque et par une société. l'éthique est en demière analyse ce qui inflige un 'démenti' au scientisme et à ses prétentions à réduire la vérité aux seuls énoncés expérimentalement vérifiés par les procédures méthodiques des sciences mathématiques de la nature.» (1991: 164) Il avance de même que l'herméneutique sut répondre au besoin d'historicité que l'hégémonie structuraliste avait précédemment effacé au profit d'une position neutre et abstraite proche du positivisme.

De Nietzsche jusqu'à Wittgenstein puis Kuhn, la pensée moderne fait écho à celte nécessaire relativisation du progrès du savoir scientifique. La traduction devrait trouver là un cadre de réflexion propice. Au sujet de la différence entre le terme traduction choisi en français à la Renaissance et l'anglais translation. Antoine Berman remarque : "Alors que la translation met l'accent sur le mouvement de transfert ou de transport. la traduction, elle, souligne plutôt l'énergie qui préside à ce transport, justement parce qu'elle renvoie à ductio et ducere. La traduction est une activité qui a un agent, aiors que la translation est un mouvement de passage plus anonyme.» (1988: 31) Si. étymologiquement. traduire signifie «faire passer» et se révèle, en ce sens, très proche de translater. il va plus loin en ce qu'il ne se connaît pas de limites. La traduction, dans l'emploi du mot qui désigne le processus et non son produit, contient et dépasse la translation. Éclairé par l'allemand Übersetzung, le terme de translation suggère un transfert, certes, mais aussi son accomplissement. La (tra)duction, elle, loi d'une dynamique des corps, langagiers pour ce qui nous conceme, $n$ "implique pas l'arrêt du processus. Sans source ni cible, sans départ ni arrivée, une traduction est toujours sur une ligne de fuite. jamais définitive, toujours ouverte à la reprise, à la retraduction, non pas sous l'effet de contingences historiques (améliorations techniques ou modifications des attentes et des sensibilités culturelles) mais par nature et nécessité. La traduction est une herméneutique parce que son inscription dans l'histoire expose en permanence l'original à, comme l'écrit HansGeorg Gadamer, «de nouvelles sources de compréhension qui révèlent des rapports de sens insoupçonnés" (1976: 139).

$\mathrm{Si}$, cependant, la traductologie choisit la linguistique comme fée tutélaire, c'est qu'elle avait besoin d'être rassurée. L'incertitude du sens est une notion que la culture occidentale redoute comme la peste et. dès les Grecs. relayés par le christianisme, elle a choisi de baliser sa pensée le long de voies fermement tracées, dogmatisme religieux ou rationalité, rejetant l'obscur et le flou dans les lointains du blasphème ou de la folie. L'Église ou le laboratoire combattent le même ennemi, l'incroyance, même si celle-ci n'est simplement qu'une façon de croire différemment, par exemple en intégrant le doute. Or l'incertitude du sens règne au cơur même de l'acte de traduire. Ce qui précisément pose problème à la pensée traditionnelle qui voit dans l'acte le produit d'une décision 
prise selon des normes et des critères. L'histoire de la morale le démontre exemplairement, ce pour quoi la pensée contemporaine, confrontée à une histoire bafouant les principes élémentaires choisis par les sociétés modernes, privilégie l'éthique sur la morale. La traduction joue en effet sur un sens incertain. puisqu'elle flotte entre deux langues, au risque d'ailleurs de la folie dont Hölderlin traducteur demeure le tragique exemple. Walter Benjamin dit de ses traductions de Sophocle : “|...l l'immense danger qui, dès le départ, guette toute traduction : que les portes d'un langage si élargi et si dominé retombent et enferment le traducteur dans le silence. $|\ldots|$ lci le sens s'effondre d'abîme en abìme, jusqu à risquer de se perdre dans les gouffres sans fond du langage». (1971 : 275) La folie, au demeurant, ne réside pas tant dans l'impossibilité pour le sujet de traduire correctement son rapport au monde, dans les deux directions - ce qu'il reçoit du monde. ce qu'il y exprime -, mais dans l'impossibilité de choisir une version parmi

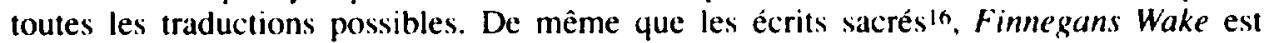
impossible à traduire parce que l'ouvre est constituée de cette impossibilité. C'est aussi cette impossibilité qui incite à traduire et qu il faut traduire.

Traduisant, je mets au jour la non-adéquation d'un sens et d'une forme, d'une signification et d'un langage. alors que je fonde la possibilité même du traduire sur cet écart. Je ne peux jamais maîtriser l'ensemble des paramètres qui cernent et fixent le sens d'un texte dont on sait que le «message» n'est qu'une partie. Par ailleurs. en quelle langue com-prendre un texte, le prendre avec moi, en moi? En langue de départ ou en langue d'arrivée "? Est-ce que je ne le comprends pas uniquement, pour citer Derrida. dans la differancé, dans le retardement? Je comprends l'original rédigé en langue de départ déjà en langue d'arrivée avant même que de le traduire en celle-ci. à moins que je ne le comprenne dans une métalangue purement sémantisée échappant à toute formalisation grammaticale ou lexicale. ce dont rien n'est moins sûr. Je retiens ou contiens le passage, la traduction, avant de l'effectuer mais ce retard me permet la compréhension, ce retard est son essence. Je ne suis jamais sûr en dernier regard d avoir saisi le sens du texte de départ et ne suis jamais maître, au demeurant, du sens du texte d'arrivée qui, pour les mêmes raisons, est susceptible de lectures multiples. Polysémie au départ, polysémie à l'arrivée. le sens n'est pas incertain : il est introuvable. Ou, pour reprendre un terme freudien - et le rapprochement $n$ 'est pas fortuit - il est inachevable ${ }^{17}$. Seul le rapport au sujet dans son historicité permettra la saisie de la signifiance. L'herméneutique désigne le lieu de la traduction comme lieu intermédiaire : la position du traducteur $n$ 'est jamais ni d'un côté ni de l'autre. II n'est soumis ni aux exigences du sens originel dépendant de son historicité comprise comme fixation, ni des obligations socioculturelles appelant les conditions d'énonciation du texte d'arrivée. La tâche du traducteur est de faire le lien. sa fonction consiste essentiellement en une médiation ${ }^{1 k}$. Son lieu est l'entre-langues et la version interlinéaire de la traduction biblique que Benjamin pose comme modèle de toute traduction en conclusion de sa «Tâche du traducteur" se comprend de cet éclairage herméneutique. De là aussi le refus de Benjamin de voir dans la traduction un acte de communication, refusant l'instrumentalité du langage. D'où l'intérêt pour la traductologie de se tourner vers les nouvelles épistémologies qui interrogent et travaillent l'incertitude du sens et de se situer parmi elles. On a parlé du linguistic turn dans les sciences humaines. Peut-être assisterons-nous au translative turn.

C'est dire que La traduction, qu' est-ce' à dire?' accueille et privilégie un faisceau d'approches diverses ${ }^{19}$ : tentatives de théorisation, réflexions spéculatives, témoignages d'une pratique, développements polémiques, élaboration esthétique... qui toutes convergent pour montrer qu en matière de théorie de la traduction, la seule cohérence possible est une co-errance. Après tout. le sourcier et le puisatier ne sont pas étrangers l'un à l'autre el même si je suis loin de partager les prémisses de ceux qui. par exemple. 
n'envisagent la traduction qu'à travers les prismes normatifs et descriptifs, la traductologie me semble devoir s'appuyer sur une épistémologie de l'ouverture, ennemie d'un savoir totalisant ${ }^{20}$, répondant à la pensée de la traduction comme dialogue (ce par quoi Celan définissait aussi le poème). La traductologie s'avance alors comme modèle de l'interdisciplinarité. mesure d'une connaissance pour et de notre temps. La multiplicité signifiante que révèle tout processus traductif rencontre la multiplicité des sens attibuables à ce processus et la traduction, facteur de transformation, vecteur d'altérité dont l'essence est d'être toujours en devenir et dans un entre-deux, demeure, au ciel de notre culture, cet "obscur objet du désir (épistémologique)», un objet véritablement"1 never identifiable.

\section{Notes}

1. C'est ce que suggère Paul Ricceur en proposant le «modèle de la traduction" comme une des inspirations possibles pour concilier identité et altérite dans I'Europe à venir («Quel ethos nouveau pour I'Europe ?". Imaginer l'Europe, sous la dir. de P. Koslowski, Paris, Cerf. 1992, p. 1009). De son côté, et sur le même probleme, Umberto Eco declarait : «ll faut placer notre esperance dans une Europe polyglotte [..... une Europe de traducteurs (... I." (Le Monde, 29 sept. 1992).

2. C'est le lieu de remercier les collaborateurs qui $m$ 'ont fait l'amitié et la confiance d'exposer leurs opinions. fussent-elles divergentes des miennes, le professeur André Clas, directeur de Mera qui a eu la générosité d'accueillir ce projet éditorial difficile et enfin Danielle Collignon dont l'aide ne fut pas seulement materielle. Mes propres hypothèses sont développées dans le eadre d'une recherche subventionnée par le CRSH.

3. On comprendra qu'utilisant le terme au pluriel dans le titre du present numero, je l'entends comme attitude philosophique, méthode de pensée. m'écartant du seul héritage husserlien pour considérer la phénoménologie comme discursivité, au sens de Foucault lorsqu'il l'oppose à scientificité.

4. Dans un premier temps. je choisissais comme second póle métaphorique la figure de l'archéologue pour la dynamique de creusement el de révélation qui est la sienne. Je l'abandonnai par souci de cohérence séman. tique et par attachement à son utilisation dans la pensée freudienne dont je suis proche, alors que la logique du puisatier n'est pas la mienne.

5. Étranges voies de la rhétorique associative qui, du ciel de cendres de Celan. me faisait glisser, par ces images hydrologiques, à celui de Pagnol

6. «L'allegorie chex. Walter Benjamin, une aventure juive», Walter Benjamin et Paris, Paris, Cerf. 1986. p. 709 .

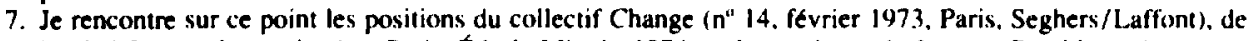
Michel Serres (La traduction. Paris, Ed. de Minuit, 1974) et les analyses de Jacques Derrida (voir notamment Positions, Paris, Éd. de Minuit, 1972. p. 31 J.

8. Pour Celan. le poème est "événement, mouvement, marche. $[\ldots]$ tentative de trouver une direction" («Discours de Brême», Paul Celan, Poèmes (tr. par J. E. Jackson), Le Muy, Ed. Unes, 1987, p. 16).

9. $\alpha$ Die Übersetzung ist die Überführung der einen Sprache in die andere durch ein Kontinuum von Verwandlungen. Kontinua der Verwandlung, nicht abstrakte Gleichheits- und Ähnlichkeitsbezirke durchmiBt die Übersetzung.m: "Über die Sprache überhaupt und über die Sprache des Menschen”, Ange'lus Novius, Ausgewählie Schrifien 2. Suhrkamp Taschenbuch. Frankfun am Main. 1966, p. 20. C"est parce que la traduction est transformation, champ de résonance que traversent toutes sones de textualités - ce que dit precisement Benjamin - que j'ai employé les termes de "transfert" el de "métamorphose" en écho a leur emploi chez Freud el Kafka.

10. Voir mon anticle "Ceci n'est pas une traduction ou Le péché de Babel", Discours social/Social Discourse. Montréal, vol. $5:$ 3-4, été-automne 1993.

11. Critique peut etre pris au sens d'analytique, ce que proposait Antoine Berman et qu'il développa dans son ouvrage posthume Pour une critique des traductions: John Donne. Paris. Gallimard. 1995. Dans un sens plus large. Henri Meschonnic en appelle à cune Theorie critique de la traduction. qui est nécessairement une partie d'une theorie critique du langage. de l'histoire el de leurs rappons" (Jona et le signifiant errant. Paris. Gallimard. 1981. p. 30). Passant de Francfort (École de) à Königsberg. on pourrait aussi songer à une Critique de la raison traductive qui s" incarnerait en une éthique et une esthétique de la traduction.

12. Voir sur ce point Nouss, 1995.

13. Cela sur la base d'une idé de la traduction comme annexion, selon l'expression de Meschonnic, ce que Nietzsche disait déjà a propos des Romains : "Autrefois, c'était conquerir que de traduire |....." (Le gai savoir, Folio / Essais, 1985, p. 110). 
14. Voir sur ce point le livre de Sherry Simon, Le Irafic des langues. Tradition et culture dans la littérature québésuse. Bureal, 1994 , et Lawrence Venutti, The Tramslafor's Imisihility. Routledge. 1994. Inutile de rappeler la place de lípresure de lémanger d'Antoine Berman dans cette reconsidération de la fonction traductive.

15. S'il faut légitimer une telle référence, je m'atutoriserai du livre de Derrida. Spéctre's de Marx (Galilé 1993). où il reinterroge le marxisme dans la perspective d'une "hantologie». In fantôme ou mieux un revenant. n'est-ce passe que l'original est prour sa traduction? I a traduction : objet fantome ou OVNI.

16. Voir mes articles "L'interdit et l'inter-dit : la traduisibilté et le sacrte», TTR, vol. 2, n" 1, 1989. et «Babel : avani, apres", $T T R$, vol. 3, n" 2, I900

17. Il est Joisible de dresser dans une perspective freudienne une analogic entre le rappon de l"original et de la traduction et le lien inconscient-conscient. Dans les deux cass. les deux discours s'énoncent dans un rappon de circularite et non de causaloté linéaire.

Sur les rapports entre phychanalyse et traduction. voir notamment Me'ta, vol. 27, n" I. 1982, et L'écrit dis temps, n"7.1984.

18. L'herméneutique gadamérienne est toute façonnée du rôle médiateur de l'interprète.

19. Justifiant ce que Régine Robin entendit lorsque je m'entretins téléphoniquement avec elle de ce projet : ،La traduction. caisse a dire ?" Jean-René l.admiral poursuivant ce jeu de signifiants par le titre de son article. A cela, il n'y a rien a redire (autre synonymic pusible, sans peoration. pour traduire). (e jaillissement créatif prouverait encore que la traduction trouve naturellement son habitat sémantique du côté de la source.

20. Parfois sous la guise d une connalssince globalisante. ce qu'on désgne en anglais par "integrated".

21. Véritablement : c'est là sal vérité.

\section{REFERENCFS}

BARTHES, Roland (197.3) : le plaisir du tekte, Paris, Seuil, coll. "Ponts".

BENJAMIN. Walter (1971): "La tâche du traducteur", (Euvres, t. 1, Mvthe "' violence, tr. M. de Gandillac. Paris, Denoxil.

BERMAN. Antoine (1988) : "De la translation a la traduction", $T J R$, vol. $1, n$ " 1.

GADAMER, Hans-(ieorg (1976): Herifé ef mithode. tr. É. Sacre, Paris, Scuil.

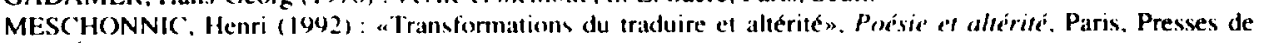
I'Ecole Normale Supériezure.

NOUSS. Alexis (1995): Lat medernite, Paris, PUF, coll. "Que sais-je?".

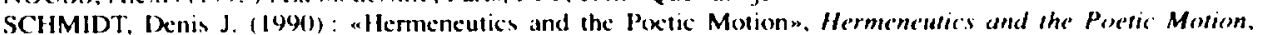
Translation Perspectives V. Center for research in Translation, State University of New York at Binghamton, pp. 1-9.

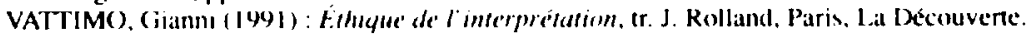

